\title{
Mental Health Care and Children Wellbeing in Ethiopia
}

\author{
Liranso G.Selamu* and Mohan S.Singhe
}

$\mathrm{PhD}$ Scholar, Department of social work at Mangalore University, India

${ }^{*}$ Corresponding author: Liranso G.Selamu, PhD Scholar, Department of social work at Mangalore University, India, E-mail: liranso.2006@gmail.com

Citation: Liranso G.Selamu, Mohan S.Singhe (2017) Mental Health Care and Children Wellbeing in Ethiopia. J Neurol Neurol Disord 3(1): 104. doi: 10.15744/2454-4981.3.104

Received Date: January 07, 2017 Accepted Date: March 08, 2017 Published Date: March 13, 2017

\begin{abstract}
Children are our future, and what happens to them affect the whole community development. The study was conducted in child caring organizations in Hossana, Ethiopia. Caregivers were used as data sources to assess the children mental health wellbeing. Univariate and bivariate analyses and Chi-square tests were used to describe the study results in a scientific manner. Results indicate having mental health problems increased the risk of a new maltreatment report and decreased the likelihood of reaching permanence. The findings highlight the children need in the welfare systems to have a prompt mental health assessment and adequate services. Policies implications include assessing necessitate of the child mental health intake that coordinates in the caring organizations have to be strengthened.
\end{abstract}

Keywords: Children Wellbeing; Permanency; Mental Health; Abused Children

\section{Introduction}

Mental health wellbeing is an essential part of children's overall health has a complex interactive relationship with their physical health and their ability to succeed in school, at work, and in society. Both physical and mental health affects how we think, feel and act on the inside and outside. For instance, an overweight young boy who is teased about his weight may withdraw socially and become depressed and may be reluctant to play with others or exercise, which further contributes to his poorer physical health and as a result poorer mental health. These issues have long-term implications on the ability of children and youth to fulfil their potential as well as consequences for the health, education, labour and criminal justice systems of our society [1].

Moreover, the high prevalence of mental health problems among children in child welfare and the inadequate provision of mental health services for this subpopulation, a consensus is emerging in the field that better coordination between child-serving agencies is needed to improve access to the service. And well-being is the ultimate goals of child welfare system for abused and neglected children. They are not only key tasks for child welfare professionals but also the main outcome measures by which policy makers often track the performance of the child welfare system. Abuse, neglect, and impermanence (e.g., lengthen stays in foster care and frequent placement changes) adversely affect children's psychosocial development [2].

Although many child welfare children have mental health problems, the insufficient provision of mental health services for these emotionally disturbed children has been well documented [3]. Inadequate organizational relationships between child welfare and mental health providers may partly account for the substantial gap between needs and use of mental health services. Children entering child welfare often have special needs such as mental health problems [3].

In general, researchers included both physical and mental health problems have found that children with disabilities are at a higher risk of being abused and neglected than children without disabilities [4]. Children with mental health problems may be at particularly high risk because of difficulty interacting with caregivers. For instance, children with conduct disorders tended to contribute to family conflict and aggression. This finding implies that outcomes need to be examined separately for children with mental health problems. Previous studies have found three types of factors to be related to the reoccurrence of maltreatment and permanence arrangements. Thus, the objective of this study was to compare safety and permanence outcomes of children with mental health needs from those of children without mental health needs.

An estimated 15 million of our nation's young people can currently be diagnosed with a mental health disorder. Much more are at risk of developing a disorder due to risk factors in their biology or genetics; within their families, schools, and communities; 
and among their peers. There is a great need for mental health professionals to provide the best available care based on scientific evidence, good clinical expertise, and that takes into account the unique characteristics of the child or adolescent. However, it is estimated that only about 7 percent of these youth who need services receive appropriate help from mental health professionals $[5]$.

Only two studies to date have examined the effects of children's mental health needs on repetitive maltreatment [6]. One study found that infants' mental health problems increased the risk of maltreatment reoccurrence. The very small number of children with either mental health problems or mental retardation, however, was very small. Thus, their inference is somewhat problematic. Another study did not find any association between the presence of a DSM diagnosis and recurrence in the first 60 days among 174 families with children returning home from their first stay in substitute care [7]. Although a few studies investigated maltreatment reoccurrence through multiple time intervals no previous study has examined the role of mental health needs in maltreatment recurrence across both short and long periods. Such a design is necessary to support policy decisions addressing children's longterm safety.

Overall, prior research indicates that the mental health of children in the child welfare system may affect safety and probably affects permanence. Some limitations of previous studies, however, merit additional investigation. First, the two studies on mental health and safety had conflicting results. Neither did a clear pattern emerge from the most substantial body of evidence concerning permanence. Different samples and varying follow-up intervals may account for inconsistent findings. Thus, it is necessary to conduct research that examines permanence over multiple periods of time within one population [1].

Given the rising levels of emotional and financial stress for families with children suffering from mental health problems, these children have elevated risks of repeated maltreatment. Potential caregivers may be concerned about meeting these children's special needs. Caseworkers may also regard children's mental health problem reasons for being removed from home. Thus, children with mental health needs may be less likely to reach permanence than those without such needs. Formally, the hypothesized: children with mental health needs are more likely to experience reoccurrence of maltreatment compared to those without mental health needs. And, children with mental health needs are less likely to achieve permanence compared to those without mental health needs.

On the other vein, the majority of children with mental health problems go untreated, and the gap between need and service use is assumed to be wider in rural than in urban areas. It is also assumed that rural families of children with mental health problems experience a greater financial and emotional impact than urban families. These assumptions reflect the lower availability of mental health specialty care and support services in rural areas. Lower income and more limited economic opportunities may further hamper the ability of rural families to care for children with more severe mental health problems. Therefore, the objective of the study was to compare safety and permanence outcomes of children with mental health needs from those of children without mental health needs in two child caring organizations in Hossana, Ethiopia.

\section{Methods}

Data were drawn from the two child protective service organizations. The organizations were working on child welfare and family well-being which is experienced with community empowerment, and other services. The target respondents of the investigation include all children in the organizations who were exposed for abused. A stratified design was used to fix 162 sample children per organizations within four months after the close of the assessment. Data from whole study symbols were used in the analyses. In addition, to maintain reliability with child welfare outcomes: annual report of the organizations. Proportional danger modelling was also used to estimation the belongings of mental health needs on likelihood of eventual durability and reunification.

Univariate and Bivariate analyses were used to depict and compare subgroups (e.g., children with a new maltreatment report vs. children without new reports, children who achieved permanence vs. children who did not achieve permanence). Chi-square tests were used to resolve on whether children's description in groups. Numerous imputations were used to attribute missing independent variable standards. Finally, proportional hazard models and generalized linear models with poison distributions were used to test the discover hypotheses. Proportional hazard modeling approximated the belongings of children's mental health needs on wellbeing. Because of the multilevel structure of data, cluster option was used in models to specify that the observations were independent across child welfare organizations.

\section{Results}

The objective of the study was to compare safety and permanence outcomes of children with mental health needs from those of children without mental health needs in two child caring organizations in Hossana, Ethiopia.

In Table 1: an overview of age and sex background information on the study participants. In all, there were two hundred twenty respondents participated in the research. With respect to age level, 114 (53\%) were 0-5 age ranges, 57 (26\%) were 6-10 age ranges, and $49(21 \%)$ were 11-14 age ranges. Out of this number $162(73 \%)$ were males, and $58(27 \%)$ were females. 


\begin{tabular}{|c|c|c|c|c|c|c|}
\hline \multirow{2}{*}{$\begin{array}{c}\text { Age } \\
\text { Level }\end{array}$} & \multicolumn{2}{|c|}{$0-5$} & \multicolumn{2}{c|}{$6-10$} & \multicolumn{2}{c|}{$11-14$} \\
\cline { 2 - 7 } & No & $\%$ & No & $\%$ & No & $\%$ \\
\cline { 2 - 7 } & 114 & 53 & 57 & 26 & 49 & 21 \\
\hline \multirow{3}{*}{ Sex } & \multicolumn{4}{|c|}{ Male } & \multicolumn{2}{c|}{ Female } \\
\cline { 2 - 7 } & \multicolumn{2}{|c|}{ No } & \multicolumn{2}{|c|}{$\%$} & No & $\%$ \\
\cline { 2 - 7 } & \multicolumn{2}{|c|}{162} & \multicolumn{2}{|c|}{73} & 58 & 27 \\
\hline
\end{tabular}

Table 1: An overview of age and sex background information of the respondents

The prevalence of mental health needs and reoccurrence of maltreatment and total behavior problems within the safety sample was $41.3 \%$; that of externalizing pattern behaviors was $38.4 \%$, and that of internalizing pattern behaviors was $20.3 \%$. In the first 6 months after the child welfare organizations began their study (183 days), only $4 \%$ of children had a fresh report. The length of time from the investigation date to the date of the later report ranged from 0 to 181 days (mean=67.4, SD=6.4). From the date, the investigation was conducted to the end of Sign 4, over one-quarter of children (27.6\%) had at least one new maltreatment report. The length of time from the investigation date to the date of the subsequent report ranged from starting date to ending date $(\mathrm{SD}=31.8)$. For those without any maltreatment report, the period of observation ranged from 172 to 460 days. This discrepancy can be explained by the initial maltreatment investigation beginning at a different time, as well as a difference in the timing of the Sign 4 interview.

\section{Bivariate Safety Analyses}

Chi-square tests indicated that differences did exist in the total behavior problem and externalizing behavior scales, family risk level, annual family income, childhood chronic disease, and annual family income. The externalizing behavior status of re-abused children significantly differed from that of children without such practice, both in the short run $\left(\chi^{2}=8.97, p=0.002\right)$ and in the long run $\left(\chi^{2}=4.49, \mathrm{p}=0.022\right)$. For children who had externalizing behaviors in a clinical range $(>53)$, the likelihood of a new maltreatment report in the first 6 months was nearly 3 times that of children who did not have externalizing behavior $(5.2 \%$ vs.1.6\%). By the end of model 4, the proportions had risen to $20.1 \%$ and $12.1 \%$, respectively. In the short-term, children with whole behavior problems at baseline was also almost 2.7 times more possible to have a new description than children who did not have total behavior problems $\left(6.0 \%\right.$ vs.2.6\%; $\left.\chi^{2}=5.46, p=0.013\right)$. The proportion of internalizing pattern behaviors, however, was similar between groups $(\mathrm{p}>0.05)$.

Three proportional risk models were used to evaluate the things of mental health needs on safety in the first six months after a research. Model 1 included total behavior problems as the independent variable; model 2 examined externalizing behaviors, and model 3 identified associations between internalizing behaviors and re-abuse. Several factors were significantly related to the reoccurrence of maltreatment. First, the externalizing score was an important forecaster of maltreatment reappearance. The maltreatment recurrence risk among children with externalizing behaviors at baseline was $82 \%$ higher than that of others ( $\mathrm{p}<0.05$ ). Second, older children had less likelihood of re-abuse than younger ones in the first 6 months after investigations. For example, the danger of being re-maltreated among children aged from 6-10 years old was $62 \%(\mathrm{p}<0.01)$ lower than those $2-5$ years old, Model 1. Third, children in foster care were more likely to have a new maltreatment report than those living in an in home setting risk relation $=4.39, \mathrm{p}<0.05$; Model 2. Finally, children with insurance status other than Medicaid (e.g., self-pay) had a lower probability of incurring a new maltreatment report (risk relation $=0.15, \mathrm{p}<0.05$; Model 1 ).

While forecasting long phrase safety, the same three indicators of mental health needs were then examined for their relations with re abuse in the long term models. The total manners problem score was used as an independent variable in Model 4, externalizing behaviors in Model 5, and internalizing behaviors in Model 6. Both total behavior problems and externalizing behavior problems were significantly connected to the recurrence of mistreatment. Children with total behavior problems were $43 \%$ more likely to experience re-abuse than children with a normal or borderline total CBCL score $(\mathrm{p}<0.001)$. The risk of being abused once again was $54 \%$ higher for children with clinical externalizing behavior scores $(\mathrm{p}<0.001)$. However, children with internalizing behaviors did not show a higher danger of mistreatment recurrence than those without internalizing behavior $(\mathrm{p}>0.05)$.

There were also some significant predictors among the controls. Children whose maltreatment type at baseline was neglect were 2.61 times more likely to have a new maltreatment report than children whose maltreatment type at baseline was physical maltreatment $(\mathrm{p}<0.05)$ (Model 4). Sexual abuse was also linked with a greater hazard of re-abuse $(\mathrm{p}<0.05)$. Children staying in group homes at baseline had a $76 \%$ lower risk of re-abuse than children at home $(\mathrm{p}<0.001)$ (Model 4). Children with an insurance status other than Medicaid (e.g., self-pay) had a lower likelihood of incurring a new maltreatment report by model 4 (risk ratio=0.56, $<<0.05$; Model 4). Finally, children with high-risk levels had a 77\% higher chance of incurring a new maltreatment report than children with low-risk levels (risk ratio $=1.77, \mathrm{p}<0.05$; Model 6).

The permanence of the children, the prevalence of mental health needs and prevalence of permanence outcomes was stated as follows. The prevalence of total behavior problems and externalizing behaviors among children in organizational support at baseline were $64.1 \%$ and $34.4 \%$, respectively, while the prevalence of internalizing pattern behaviors was only $34.1 \%$. Overall, the 
length of time in foster care for the permanence sample ranged from 172 to 460 day (mean=77.1, SD=33.8). At the end of Sign 4, 45.7\% of children had achieved either reunification adoption, or legal guardianship. The average length of time to eventual permanence among those children was 258.7 days ( $\mathrm{SD}=29.6)$. In addition, nearly a quarter of children returned to their homes by Sign $4(24.0 \%)$. The average length of time to achieve reunification among these children was 415.6 days $(\mathrm{SD}=44.1)$. Finally, the average number of placements was 5.0, with the range from 1 to $12(\mathrm{SD}=0.2)$.

\section{Bivariate Analysis}

The study compares the demographic, socioeconomic, and child welfare service characteristics at baseline between children who did and did not achieve permanence by Sign 4 . The bivariate analysis described that away no statistically significant differences between the two groups in total behavior problems. There were, however, differences in age, insurance type, the receipt of child welfare services, and risk levels between groups with different permanence outcomes $(\mathrm{p}<0.05)$. Whereas predicting ultimate permanence, three proportional risk models were used to assess the effects of mental health needs on permanence outcomes. The independent variable was the presence of total behavior problems, in all models behavioral result. Proportional danger models revealed that none of these mental health measures was a significant forecaster of permanence among children living in organizational support at baseline ( $\mathrm{p}>0.05)$.

\section{Discussion}

This study evaluated the safety and permanence outcomes among children with mental health needs in the child wellbeing system and tests whether children with and without mental health needs differed in safety and permanence. It hypothesized that in contrast to those without such needs; children with mental health needs were at a higher risk of experiencing re-maltreatment and failing to achieve permanence (e.g., achieving reunification, adoption, legal guardianship, and having fewer out of home placements).

The results support hypotheses and externalizing pattern behaviors predicted the reoccurrence of maltreatment not only for the first 6 months but also for a period of up to 5 years. Furthermore, total behavior problems reduced the likelihood of long-term safety, whereas internalizing outline behaviors were considerably and really related to weakening to achieve reunification. More placements were significantly associated with total behavior, externalizing, and internalizing problems. In summary, the study finds that having mental health needs increased the risk of having a new maltreatment report and decreased the likelihood of reaching permanence.

In spite of the high prevalence of mental health problems among children welfare, there have been moderately sparse empirical facts about whether or how these children's service experiences and outcomes differed from those of other children. The study is the first one to explain the relationship between preliminary mental health position and following safety and permanence in a national sample. Also, our study is the first one to investigate the exceptional role of child mental health status in forecasting the reoccurrence of childhood maltreatment in different terms. Additionally, the previous studies focused on testing the separate effects of externalizing and internalizing behaviors on safety and permanence. The contribution also improves upon prior studies in terms of more appropriate methods [8]. Instead of linear regression models, generalized linear models with Poisson regression was used to test the theory that children with mental health needs would experience more out of home placements. Generalized linear models are preferable to linear regression because count variables are skewed bounded at zero, and heteroskedastic [9].

Among the children welfare system, those with mental health needs are more likely to experience maltreatment than others. Children with mental health problems may have difficulties in attaching to or interacting with caregivers [10]. These difficulties may lead to stress between the children and their caregivers that in turn increases a risk of additional neglect or abuse. For instance, compared to caregivers of normally developing children, caregivers of children with externalizing problems reported higher child-related stress and less positive feelings about parenting [11].

On the other vein, caregivers may be unwilling to take these children given the doubt over the level of care required. In a telephone survey of 916 people from a nationally representative sample, most people agreed that the relationship between adopted children with severe behavioral problems and their adoptive parents would eventually be disrupted [12]. In addition, children with severe psychological conditions are usually regarded as having special needs. This may give potential adoptive parents pause. From a caseworker's perspective, severe mental health problems may be considered a negative outcome for the family. This is most likely one of the foremost reasons for child exclusion from families [13].

Caseworkers may also either wait against reunification because of concerns about relatives' ability to meet a child's mental health needs. These arguments suggest that mental health problems may play an important role in permanence [14]. We limited our permanency analysis within children staying in foster care rather than those living in out of home placements. First, organizational care is the most popular in child welfare system. Second, the Adoption and Safety Families Act of 1997 (ASFA) (PL 105-89) stresses that an organizational care is a temporary setting and that children in foster care need to find permanency placements earlier than before. Third, the increasing number of children in an organizational care is responsible for increasing foster care costs [15-17]. Thus, it is important to find out the factors preventing permanency for an organizational children care. From both policy and 
practice perspectives, it is necessary to address the association between children with mental health needs and their resulting safety and permanence [18].

\section{Conclusion}

The study compared these outcomes for children with and without mental health needs. Longitudinal analyses drew on data from the national study of child and adolescent well-being in the country. Proportional hazard models and generalized linear models with sampling weights were used to investigate the degree to which mental health forecasted safety and permanence. Results indicate that having mental health needs increased the danger of a new maltreatment report and decreased the likelihood of reaching permanence. Externalizing and internalizing patterns had different associations with safety and permanence. These findings highlight the need for children in the child welfare system to have a punctual mental health assessment and sufficient treatment services. Policy implications include the need to consider mental health straight after intake of children and that harmonization between child welfare agency and mental health providers should be strengthened [19].

\section{References}

1. APA (2016) Children mental health; American Psychology Association 2016 report. St.NE, Washington DC. USA.

2. Jaffee E, Caspi A, Moffitt TE, Taylor A (2004) Physical maltreatment victim to antisocial child: Evidence of an environmentally mediated process. J Abnorm Psycholo 113: 44-55.

3. Hurlburt MS, Leslie LK, Landsverk J, Barth RP, Burns BJ, et al. (2004) Contextual predictors of mental health service use among children open to child welfare. Arch Gen Psychiatry 61: 1217-24.

4. Vig S, Kaminer R, (2002). Maltreatment and developmental disabilities in children. J Dev Phy Disabil 14: $371-386$.

5. Tolan PH, Dodge KA (2005) Children's Mental Health as a Primary Care and Concern; a System for Comprehensive Support and Service. Am Psychol 60: 60114.

6. Fuller T, Wells SJ (2003) Predicting maltreatment recurrence among CPS cases with alcohol and other drug involvement. Child Youth Serv Rev 25: 553-69.

7. Fuller T, Paneth (2005) Child safety at reunification: A case-control study of maltreatment recurrence following return home from substitute care. Child Youth Serv Rev 27: 1293-306.

8. Newton L, Litrownik AJ, Landsverkc JA (2000) Children and youth in foster care: Disentangling the relationship between problem behaviours and number of placements. Chil Abu Negl 24: 1363-74.

9. McCullagh P, Nelder JA (1983) Generalized Linear Models: Chapman and Hall, Inc. London, England.

10. Fonagy P (2000) Attachment and borderline personality disorder. J Am Psy Asso, 48: 1129-46.

11. Anastopoulos, Guevremont DC, Shelton TL, DuPaul GJ (1992) Parenting stress among families of children with attention deficit hyperactivity disorder. J Abnorm Child Psychol 20:503-20.

12. Halfon N, Inkelas M, Flint R, Shoaf K, Zepeda A et al. (2002) Assessment of factors influencing the adequacy of health care services to children in foster care. Hollingsworth.

13. Thieman A A, Dail PW, (1997) Predictors of Out-of-Home Placement in a Family Preservation Program: Are Welfare Recipients Particularly Vulnerable? Pol Stu J 25: 124-39.

14. Landsverk J, Davis I, Ganger W, Newton R, Johnson I (1996) . Child Youth Serv Rev 18: 447-62.

15. Ehrle J, Scarcella CA, Geen R (2004) Teaming up: collaboration between welfare and child welfare agencies since welfare reform. Child Youth Serv Rev 26: 265-85.

16. Braungartrieker J, Rende RD, Plomin R, Defries J, Fulker D (1995) Genetic Mediation of Longitudinal Associations between Family Environment and Childhood Behaviour Problems. Dev Psychopathol 7: 233-45.

17. Clausen E (1998) Mental health problems of children in foster care. J Child Family Stud 7: 283-96.

18. Frenkel, Harmon RJ (1992) Depressed mothers: They don't always look as bad as they feel. J Am Acad Child Adolesc Psychiatry 35: 289-98.

19. Kupsinel MM, Dubsky DD (1999) Behaviourally impaired children in out-of-home care. Child Welfare 78: $297-310$.

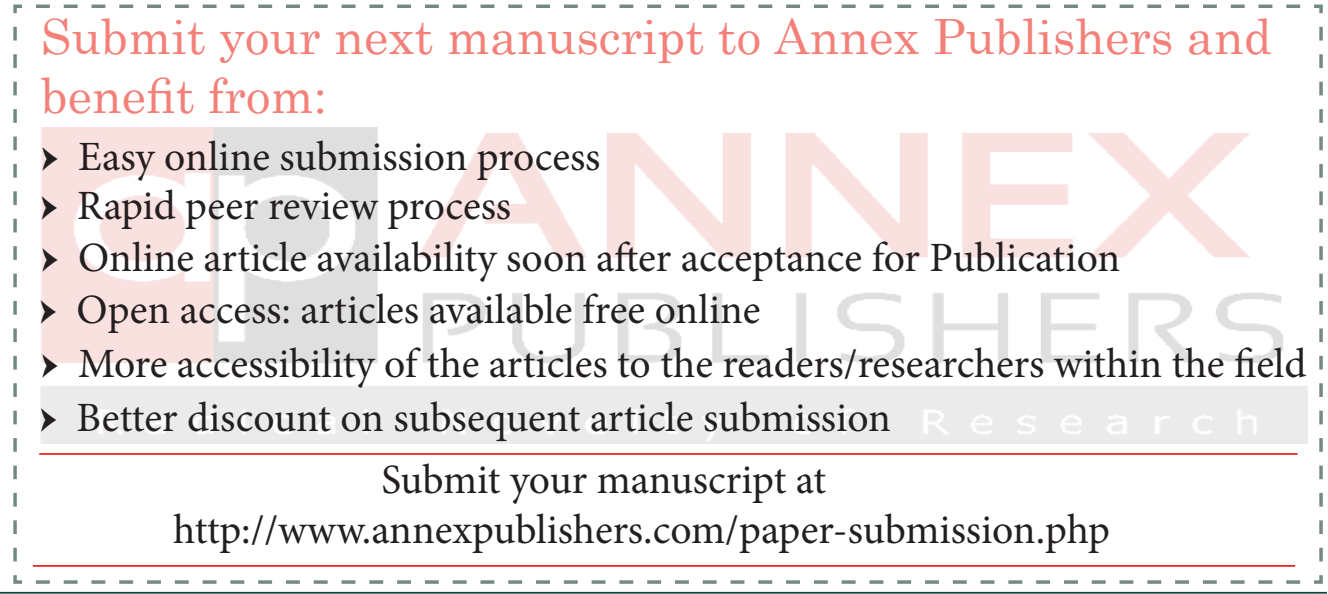

